\title{
Some Identities Involving Chebyshev Polynomials
}

\author{
Xiaoxue Li \\ School of Mathematics, Northwest University, Xi'an, Shaanxi 710127, China \\ Correspondence should be addressed to Xiaoxue Li; lxx20072012@163.com
}

Received 18 December 2014; Accepted 18 March 2015

Academic Editor: Fabio Tramontana

Copyright (C) 2015 Xiaoxue Li. This is an open access article distributed under the Creative Commons Attribution License, which permits unrestricted use, distribution, and reproduction in any medium, provided the original work is properly cited.

\begin{abstract}
The main purpose of this paper is using the combinatorial method and algebraic manipulations to study some sums of powers of Chebyshev polynomials and give several interesting identities. As some applications of these results, we obtained several divisibility properties involving Chebyshev polynomials.
\end{abstract}

\section{Introduction}

For any integer $n \geq 0$, the famous Chebyshev polynomials of the first and second kind $T_{n}(x)$ and $U_{n}(x)$ are defined as follows:

$$
\begin{aligned}
& T_{0}(x)=1, T_{1}(x)=x, \text { and } T_{n+1}(x)=2 x T_{n}(x)- \\
& T_{n-1}(x) \text { for all } n \geq 1 ; \\
& U_{0}(x)=1, U_{1}(x)=2 x, \text { and } U_{n+1}(x)=2 x U_{n}(x)- \\
& U_{n-1}(x) \text { for all } n \geq 1 .
\end{aligned}
$$

It is clear that these polynomials are the second-order linear recurrence polynomial; they satisfy the computational formulae:

$$
\begin{aligned}
T_{n}(x)= & \frac{1}{2}\left[\left(x+\sqrt{x^{2}-1}\right)^{n}+\left(x-\sqrt{x^{2}-1}\right)^{n}\right], \\
U_{n}(x)= & \frac{1}{2 \sqrt{x^{2}-1}} \\
& \cdot\left[\left(x+\sqrt{x^{2}-1}\right)^{n+1}-\left(x-\sqrt{x^{2}-1}\right)^{n+1}\right] .
\end{aligned}
$$

About the elementary properties of Chebyshev polynomials and related second-order linear recurrences, many authors had studied them and obtained a series of interesting conclusions. For example, some of the theoretical results can be found in [1-4], and other some important applications of the Chebyshev polynomials can also be found in [5-10].
Recently, several authors studied the sums of powers of Fibonacci numbers $\left\{F_{n}\right\}$ and Lucas numbers $\left\{L_{n}\right\}$, and obtained a series of important identities; see [11-13]. At the same time, Melham [13] also proposed the following two conjectures.

Conjecture 1. Let $m \geq 1$ be an integer. Then the sum

$$
L_{1} L_{3} L_{5} \cdots L_{2 m+1} \sum_{k=1}^{n} F_{2 k}^{2 m+1}
$$

can be expressed as $\left(F_{2 n+1}-1\right)^{2} P_{2 m-1}\left(F_{2 n+1}\right)$, where $P_{2 m-1}(x)$ is a polynomial of degree $2 m-1$ with integer coefficients.

Conjecture 2. Let $m \geq 0$ be an integer. Then the sum

$$
L_{1} L_{3} L_{5} \cdots L_{2 m+1} \sum_{k=1}^{n} L_{2 k}^{2 m+1}
$$

can be expressed as $\left(L_{2 n+1}-1\right) Q_{2 m}\left(L_{2 n+1}\right)$, where $Q_{2 m}(x)$ is a polynomial of degree $2 m$ with integer coefficients.

Wang and Zhang [14] solved the Conjecture 2 completely and made some substantial progress for the Conjecture 1.

The main purpose of this paper is using the algebraic manipulations to obtain some identities involving Chebyshev polynomials of the first and second kind $T_{n}(x)$ and $U_{n}(x)$. As some applications, we give three interesting corollaries. That is, we will prove the following two results. 
Theorem 3. For any positive integers $h$ and $n$, we have the identities

(a)

$$
\sum_{m=0}^{h} T_{2 m+1}^{2 n+1}(x)=\frac{1}{2^{2 n+1}} \sum_{k=0}^{n}\left(\begin{array}{c}
2 n+1 \\
n-k
\end{array}\right) \frac{U_{2(2 k+1)(h+1)-1}(x)}{U_{2 k}(x)} ;
$$

(b)

$$
\begin{aligned}
\sum_{m=0}^{h} U_{2 m}^{2 n+1}(x)= & \frac{1}{4^{n}\left(x^{2}-1\right)^{n}} \\
& \cdot \sum_{k=0}^{n}\left(\begin{array}{c}
2 n+1 \\
n-k
\end{array}\right)(-1)^{n-k} \frac{U_{(2 k+1)(h+1)-1}^{2}(x)}{U_{2 k}(x)} .
\end{aligned}
$$

Theorem 4. For any positive integers $h$ and $n$, we have the identities

(A)

$$
\begin{aligned}
& \sum_{m=1}^{h} T_{2 m}^{2 n+1}(x) \\
& \quad=\frac{1}{2^{2 n+1}} \sum_{k=0}^{n}\left(\begin{array}{c}
2 n+1 \\
n-k
\end{array}\right) \frac{U_{(2 k+1)(2 h+1)-1}(x)-U_{2 k}(x)}{U_{2 k}(x)}
\end{aligned}
$$

(B)

$$
\begin{aligned}
\sum_{m=1}^{h} U_{2 m-1}^{2 n+1}(x) & \\
= & \frac{1}{2^{2 n+1}\left(x^{2}-1\right)^{n+1}} \\
& \cdot \sum_{k=0}^{n}\left(\begin{array}{c}
2 n+1 \\
n-k
\end{array}\right)(-1)^{n-k} \frac{T_{(2 k+1)(2 h+1)}(x)-T_{2 k+1}(x)}{U_{2 k}(x)}
\end{aligned}
$$

The benefit of these identities is that it can transform the complex sums of powers of Chebyshev polynomials that become relatively simple linear sums of Chebyshev polynomials. This can simplify the calculation problems related to the sums of powers of Chebyshev polynomials.

Whether there exists an exact expression for the derivative or integral of the Chebyshev polynomials of the first kind in terms of the Chebyshev polynomials of the first kind (and vice-versa) is an open problem. we will be looking for some new methods to further research.

Note that $T_{n}(\cos \theta)=\cos (n \theta)$ and $U_{n}(\cos \theta)=\sin ((n+$ 1) $\theta) / \sin \theta$; it is clear that from Theorems 3 and 4 we can deduce some identities involving $\sin x$ and $\cos x$. On the other hand, we can also obtain some divisibility properties involving Chebyshev polynomials. That is, we have the following.
Corollary 5. Let $h \geq 1$ and $n \geq 0$ be two integers. Then the sum

$$
U_{0}(x) U_{2}(x) U_{4}(x) \cdots U_{2 n}(x) \sum_{m=0}^{h} T_{2 m+1}^{2 n+1}(x)
$$

can be divided by polynomials $U_{2 h+1}(x)$.

The sum

$$
U_{0}(x) U_{2}(x) U_{4}(x) \cdots U_{2 n}(x) \sum_{m=0}^{h} U_{2 m}^{2 n+1}(x)
$$

can be divided by polynomials $U_{h}^{2}(x)$.

Corollary 6. Let $h \geq 1$ and $n \geq 0$ be two integers. Then the sum

$$
U_{0}(x) U_{2}(x) U_{4}(x) \cdots U_{2 n}(x) \sum_{m=1}^{h} T_{2 m}^{2 n+1}(x)
$$

can be expressed as $\left(U_{2 h}(x)-1\right) P_{2 n}\left(x, T_{2 h+1}(x)\right)$, where $P_{2 n}(x, y)$ is an integer coefficients polynomial of two variables with degree $2 n$ of $y$.

Corollary 7. Let $h \geq 1$ and $n \geq 0$ be two integers. Then the sum

$$
U_{0}(x) U_{2}(x) U_{4}(x) \cdots U_{2 n}(x) \sum_{m=1}^{h} U_{2 m-1}^{2 n+1}(x)
$$

can be expressed as $\left(T_{2 h+1}(x)-x\right) Q_{2 n}\left(x, T_{2 h+1}(x)\right)$, where $Q_{2 n}(x, y)$ is an integer coefficients polynomial of two variables with degree $2 n$ of $y$.

\section{Proof of the Theorems}

In this section, we will use the algebraic manipulations to complete the proof of our theorems. First we prove Theorem 3. In fact, for any positive integer $n$ and real number $y \neq 0$, by using the familiar binomial expansion

$$
\left(y+\frac{1}{y}\right)^{n}=\sum_{k=0}^{n}\left(\begin{array}{l}
n \\
k
\end{array}\right) y^{n-2 k}
$$

we may get

$$
\begin{gathered}
\left(y+\frac{1}{y}\right)^{2 n+1}=\sum_{k=0}^{n}\left(\begin{array}{c}
2 n+1 \\
n-k
\end{array}\right)\left(y^{2 k+1}+\frac{1}{y^{2 k+1}}\right), \\
\left(y-\frac{1}{y}\right)^{2 n+1}=\sum_{k=0}^{n}\left(\begin{array}{c}
2 n+1 \\
n-k
\end{array}\right)(-1)^{n-k}\left(y^{2 k+1}-\frac{1}{y^{2 k+1}}\right) .
\end{gathered}
$$

Now we take $y=\left(x+\sqrt{x^{2}-1}\right)^{2 m+1}$ in (13); then note that $1 / y=\left(x-\sqrt{x^{2}-1}\right)^{2 m+1}$; from the definitions of $T_{n}(x)$ and $U_{n}(x)$, we may immediately deduce the identities

$$
T_{2 m+1}^{2 n+1}(x)=\frac{1}{4^{n}} \sum_{k=0}^{n}\left(\begin{array}{c}
2 n+1 \\
n-k
\end{array}\right) T_{(2 m+1)(2 k+1)}(x),
$$




$$
\begin{aligned}
U_{2 m}^{2 n+1}(x)= & \frac{1}{4^{n}\left(x^{2}-1\right)^{n}} \\
& \cdot \sum_{k=0}^{n}\left(\begin{array}{c}
2 n+1 \\
n-k
\end{array}\right)(-1)^{n-k} U_{4 m k+2 m+2 k}(x) .
\end{aligned}
$$

If we take $y=\left(x+\sqrt{x^{2}-1}\right)^{2 m}$ in (13), then we can also deduce the identities

$$
\begin{aligned}
T_{2 m}^{2 n+1}(x)=\frac{1}{4^{n}} \sum_{k=0}^{n}\left(\begin{array}{c}
2 n+1 \\
n-k
\end{array}\right) T_{2 m(2 k+1)}(x), \\
U_{2 m-1}^{2 n+1}(x)=\frac{1}{4^{n}\left(x^{2}-1\right)^{n}} \\
\cdot \sum_{k=0}^{n}\left(\begin{array}{c}
2 n+1 \\
n-k
\end{array}\right)(-1)^{n-k} U_{2 m(2 k+1)-1}(x) .
\end{aligned}
$$

Let $\alpha=x+\sqrt{x^{2}-1}$ and $\beta=1 / \alpha=x-\sqrt{x^{2}-1}$. Then for any integer $h>0$, note that $\alpha \beta=1$; from (14) and the definitions of $U_{n}(x)$ and $T_{n}(x)$ we have

$$
\begin{aligned}
\sum_{m=0}^{h} T_{2 m+1}^{2 n+1}(x) & \\
= & \frac{1}{4^{n}} \sum_{k=0}^{n}\left(\begin{array}{c}
2 n+1 \\
n-k
\end{array}\right) \sum_{m=0}^{h} T_{(2 m+1)(2 k+1)}(x) \\
= & \frac{1}{2 \cdot 4^{n}} \sum_{k=0}^{n}\left(\begin{array}{c}
2 n+1 \\
n-k
\end{array}\right)\left[\frac{\alpha^{2 k+1}\left(\alpha^{2(2 k+1)(h+1)}-1\right)}{\alpha^{2(2 k+1)}-1}\right. \\
& \left.+\frac{\beta^{2 k+1}\left(\beta^{2(2 k+1)(h+1)}-1\right)}{\beta^{2(2 k+1)}-1}\right] \\
= & \frac{1}{2 \cdot 4^{n}} \sum_{k=0}^{n}\left(\begin{array}{c}
2 n+1 \\
n-k
\end{array}\right) \frac{\alpha^{4 k h+4 k+2 h+2}-\beta^{4 k h+4 k+2 h+2}}{\alpha^{2 k+1}-\beta^{2 k+1}} \\
= & \frac{1}{2^{2 n+1}} \sum_{k=0}^{n}\left(\begin{array}{c}
2 n+1 \\
n-k
\end{array}\right) \frac{U_{4 h k+4 k+2 h+1}(x)}{U_{2 k}(x)} .
\end{aligned}
$$

This proves the identity (a) of Theorem 3 .

Similarly, from formula (15) we can deduce the identity (b) of Theorem 3.

Now we prove Theorem 4. From (16) we have

$$
\begin{aligned}
& \sum_{m=1}^{h} T_{2 m}^{2 n+1}(x) \\
&=\frac{1}{4^{n}} \sum_{k=0}^{n}\left(\begin{array}{c}
2 n+1 \\
n-k
\end{array}\right) \sum_{m=1}^{h} T_{2 m(2 k+1)}(x) \\
&=\frac{1}{2^{2 n+1}} \sum_{k=0}^{n}\left(\begin{array}{c}
2 n+1 \\
n-k
\end{array}\right)\left[\frac{\alpha^{2(2 k+1)}\left(\alpha^{2 h(2 k+1)}-1\right)}{\alpha^{2(2 k+1)}-1}\right. \\
&\left.+\frac{\beta^{2(2 k+1)}\left(\beta^{2 h(2 k+1)}-1\right)}{\beta^{2(2 k+1)}-1}\right]
\end{aligned}
$$

$$
\begin{aligned}
=\frac{1}{2^{2 n+1}} \sum_{k=0}^{n}\left(\begin{array}{c}
2 n+1 \\
n-k
\end{array}\right)\left[\frac{\alpha^{(2 k+1)(2 h+1)}-\alpha^{2 k+1}}{\alpha^{2 k+1}-\beta^{2 k+1}}\right. \\
\left.-\frac{\beta^{(2 k+1)(2 h+1)}-\beta^{2 k+1}}{\alpha^{2 k+1}-\beta^{2 k+1}}\right] \\
=\frac{1}{2^{2 n+1}} \sum_{k=0}^{n}\left(\begin{array}{c}
2 n+1 \\
n-k
\end{array}\right) \frac{U_{(2 k+1)(2 h+1)-1}(x)-U_{2 k}(x)}{U_{2 k}(x)} .
\end{aligned}
$$

This proves the identity (A) of Theorem 4 .

Similarly, from formula (17) we can also deduce the identity (B) of Theorem 4.

Now we use Theorem 3 to prove Corollary 5. From the properties of Chebyshev polynomials we know that $T_{n}\left(T_{m}(x)\right)=T_{m n}(x)$ and $U_{n}\left(T_{m}(x)\right)=U_{m(n+1)-1}(x) / U_{m-1}(x)$; from (a) of Theorem 3 we may immediately deduce that

$$
\begin{aligned}
& \sum_{m=0}^{h} T_{2 m+1}^{2 n+1}(x) \\
& \quad=\frac{1}{2^{2 n+1}} \sum_{k=0}^{n}\left(\begin{array}{c}
2 n+1 \\
n-k
\end{array}\right) \frac{U_{2 h+1}(x) \cdot U_{2 k}\left(T_{2 h+2}(x)\right)}{U_{2 k}(x)} .
\end{aligned}
$$

That is, the power sum

$$
U_{0}(x) U_{2}(x) U_{4}(x) \cdots U_{2 n}(x) \sum_{m=0}^{h} T_{2 m+1}^{2 n+1}(x)
$$

can be divided by polynomial $U_{2 h+1}(x)$.

Similarly, note that $U_{(2 k+1)(h+1)-1}^{2}(x)=U_{h}^{2}(x)$. $U_{2 k}^{2}\left(T_{h+1}(x)\right)$; from (b) of Theorem 3 we know that $U_{h}^{2}(x)$ divide the power sum

$$
U_{0}(x) U_{2}(x) U_{4}(x) \cdots U_{2 n}(x) \sum_{m=0}^{h} U_{2 m}^{2 n+1}(x) .
$$

This proves Corollary 5 .

Now we use (B) of Theorem 4 to prove Corollary 7. It is clear that if $F(x)$ is an integer coefficients polynomial with variable $x$, then, for any polynomials $a(x)$ and $b(x)$, we have $a(x)-b(x)$ divides $F(a(x))-F(b(x))$. From those properties and noting that the identity $T_{2 k+1}\left(T_{2 h+1}(x)\right)=T_{(2 h+1)(2 k+1)}(x)$ we can deduce that

$$
\begin{aligned}
& \left(T_{2 h+1}(x)-x\right) \mid T_{2 k+1}\left(T_{2 h+1}(x)\right)-T_{2 k+1}(x) \\
& \quad=T_{(2 h+1)(2 k+1)}(x)-T_{2 k+1}(x) .
\end{aligned}
$$

From (23) and (B) of Theorem 4 we can deduce that

$$
\begin{gathered}
U_{0}(x) U_{2}(x) U_{4}(x) \cdots U_{2 n}(x) \sum_{m=1}^{h} U_{2 m-1}^{2 n+1}(x) \\
=\frac{U_{0}(x) U_{2}(x) U_{4}(x) \cdots U_{2 n}(x)}{2^{2 n+1}\left(x^{2}-1\right)^{n+1}}
\end{gathered}
$$




$$
\begin{aligned}
& \cdot\left(\sum_{k=0}^{n}\left(\begin{array}{c}
2 n+1 \\
n-k
\end{array}\right)(-1)^{n-k} \frac{T_{(2 k+1)(2 h+1)}(x)-T_{2 k+1}(x)}{U_{2 k}(x)}\right) \\
= & \left(T_{2 h+1}(x)-x\right) Q_{2 n}\left(x, T_{2 h+1}(x)\right),
\end{aligned}
$$

where $Q_{2 n}(x, y)$ is an integer coefficients polynomial of two variables with degree $2 n$ of $y$. This proves Corollary 7 .

Finally, we prove Corollary 6. We first prove that

$$
\left(U_{2 h}(x)-1\right) \mid\left(U_{(2 k+1)(2 h+1)-1}(x)-U_{2 k}(x)\right)
$$

for all integers $k \geq 0$. It is clear that if $k=0$, then the conclusion is correct. So without loss of generality we can assume $k \geq 1$. Note that the identity $U_{(2 k+1)(2 h+1)-1}(x)=U_{2 h}(x)$. $U_{2 k}\left(T_{2 h+1}(x)\right)$, so we have

$$
\begin{aligned}
U_{(2 k+1)(2 h+1)-1}(x)-U_{2 k}(x)= & \left(U_{2 h}(x)-1\right) \cdot U_{2 k}\left(T_{2 h+1}(x)\right) \\
& +U_{2 k}\left(T_{2 h+1}(x)\right)-U_{2 k}(x) .
\end{aligned}
$$

Therefore, to prove (25), we only need to prove that

$$
\left(U_{2 h}(x)-1\right) \mid\left(U_{2 k}\left(T_{2 h+1}(x)\right)-U_{2 k}(x)\right) .
$$

Since $U_{2 k}(x)$ is an even function, from $(a-b) \mid\left(U_{2 k}(a)-\right.$ $\left.U_{2 k}(b)\right)$ we can deduce that $\left(T_{2 h+1}(x) \pm x\right) \mid\left(U_{2 k}\left(T_{2 h+1}(x)\right)-\right.$ $\left.U_{2 k}(x)\right)$; note that the coprime relations $\left(T_{2 h+1}(x)+x\right.$, $\left.T_{2 h+1}(x)-x\right)=x$, so from the properties of polynomials we know that

$$
\left(T_{2 h+1}^{2}(x)-x^{2}\right) \mid x \cdot\left(U_{2 k}\left(T_{2 h+1}(x)\right)-U_{2 k}(x)\right) .
$$

Since $\left(U_{2 h}(x)-1, x\right)=1$, from (28) we know that to prove (27), we only need to prove

$$
\left(U_{2 h}(x)-1\right) \mid\left(T_{2 h+1}^{2}(x)-x^{2}\right) .
$$

Note that the identity

$$
\begin{aligned}
& T_{2 h+1}^{2}(x)-x^{2} \\
&=\left(x U_{2 h}(x)-U_{2 h-1}(x)\right)^{2}-x^{2} \\
&=x^{2} U_{2 h}^{2}(x)-2 x U_{2 h}(x) U_{2 h-1}(x)+U_{2 h-1}^{2}(x)-x^{2} \\
&=x^{2}\left(U_{2 h}^{2}(x)-1\right)-U_{2 h-1}(x) U_{2 h+1}(x) \\
&=x^{2}\left(U_{2 h}^{2}(x)-1\right)-\frac{1}{4\left(x^{2}-1\right)} \\
& \\
& \cdot\left[\left(\alpha^{2 h+2}-\beta^{2 h+2}\right) \cdot\left(\alpha^{2 h}-\beta^{2 h}\right)\right] \\
&=x^{2}\left(U_{2 h}^{2}(x)-1\right)-\frac{1}{4\left(x^{2}-1\right)} \\
& \cdot\left[\left(\alpha^{2 h+1}-\beta^{2 h+1}\right)^{2}-(\alpha-\beta)^{2}\right] \\
&= x^{2}\left(U_{2 h}^{2}(x)-1\right)-U_{2 h}^{2}(x)+U_{0}^{2}(x) \\
&=\left(x^{2}-1\right) \cdot\left(U_{2 h}^{2}(x)-1\right) .
\end{aligned}
$$

From this identity we may immediately deduce (29). That is, (25) is correct.

Applying (25) and (A) of Theorem 4 we can easily deduce that the sum

$$
U_{0}(x) U_{2}(x) U_{4}(x) \cdots U_{2 n}(x) \sum_{m=1}^{h} T_{2 m}^{2 n+1}(x)
$$

can be expressed as $\left(U_{2 h}(x)-1\right) P_{2 n}\left(x, T_{2 h+1}(x)\right)$, where $P_{2 n}(x$, $y)$ is an integer coefficients polynomial of two variables with degree $2 n$ of $y$.

This completes the proofs of our all results.

\section{Conflict of Interests}

The author declares that there is no conflict of interests regarding the publication of this paper.

\section{Acknowledgments}

The author would like to thank the referees for their very helpful and detailed comments, which have significantly improved the presentation of this paper. This work is supported by the P. S. F. (2014JM1009) and N. S. F. (11371291).

\section{References}

[1] W. Zhang, "Some identities involving the Fibonacci numbers and Lucas numbers," The Fibonacci Quarterly, vol. 42, no. 2, pp. 149-154, 2004.

[2] X. Wang and D. Han, "Some identities related to Dedekind sums and the Chebyshev polynomials," International Journal of Applied Mathematics and Statistics, vol. 51, no. 21, pp. 334-339, 2013.

[3] C. Cesarano, "Identities and generating functions on Chebyshev polynomials," Georgian Mathematical Journal, vol. 19, no. 3, pp. 427-440, 2012.

[4] C.-L. Lee and K. B. Wong, “On Chebyshev's polynomials and certain combinatorial identities," Bulletin of the Malaysian Mathematical Sciences Society, vol. 34, no. 2, pp. 279-286, 2011.

[5] E. H. Doha, A. H. Bhrawy, and S. S. Ezz-Eldien, "Numerical approximations for fractional diffusion equations via a Chebyshev spectral-tau method," Central European Journal of Physics, vol. 11, no. 10, pp. 1494-1503, 2013.

[6] A. H. Bhrawy, "An efficient Jacobi pseudospectral approximation for nonlinear complex generalized Zakharov system," Applied Mathematics and Computation, vol. 247, pp. 30-46, 2014.

[7] A. H. Bhrawy and M. A. Zaky, "A method based on the Jacobi tau approximation for solving multi-term time-space fractional partial differential equations," Journal of Computational Physics, vol. 281, pp. 876-895, 2015.

[8] A. H. Bhrawy and M. A. Zaky, "Numerical simulation for twodimensional variable-order fractional nonlinear cable equation," Nonlinear Dynamics, vol. 80, no. 1-2, pp. 101-116, 2015.

[9] E. H. Doha, A. H. Bhrawy, R. M. Hafez, and M. A. Abdelkawy, "A Chebyshev-Gauss-Radau scheme for nonlinear hyperbolic system of first order," Applied Mathematics \& Information Sciences, vol. 8, no. 2, pp. 535-544, 2014. 
[10] E. H. Doha and A. H. Bhrawy, "A Jacobi spectral Galerkin method for the integrated forms of fourth-order elliptic differential equations," Numerical Methods for Partial Differential Equations, vol. 25, no. 3, pp. 712-739, 2009.

[11] H. Prodinger, "On a sum of Melham and its variants," The Fibonacci Quarterly, vol. 46-47, pp. 207-215, 2008-2009.

[12] K. Ozeki, “On Melham's sum," The Fibonacci Quarterly, vol. 4647, pp. 107-110, 2008.

[13] R. S. Melham, "Some conjectures concerning sums of odd powers of Fibonacci and Lucas numbers," The Fibonacci Quarterly, vol. 46/47, no. 4, pp. 312-315, 2009.

[14] T. Wang and W. Zhang, "Some identities involving Fibonacci, Lucas polynomials and their applications," Bulletin Mathématique de la Société des Sciences Mathématiques de Roumanie, vol. 103, no. 1, pp. 95-103, 2012. 


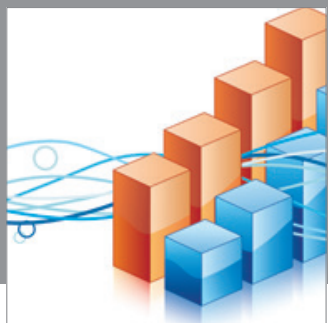

Advances in

Operations Research

mansans

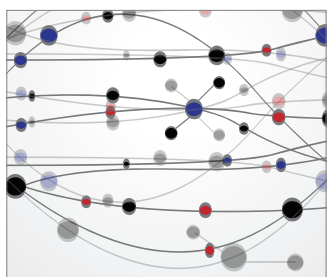

The Scientific World Journal
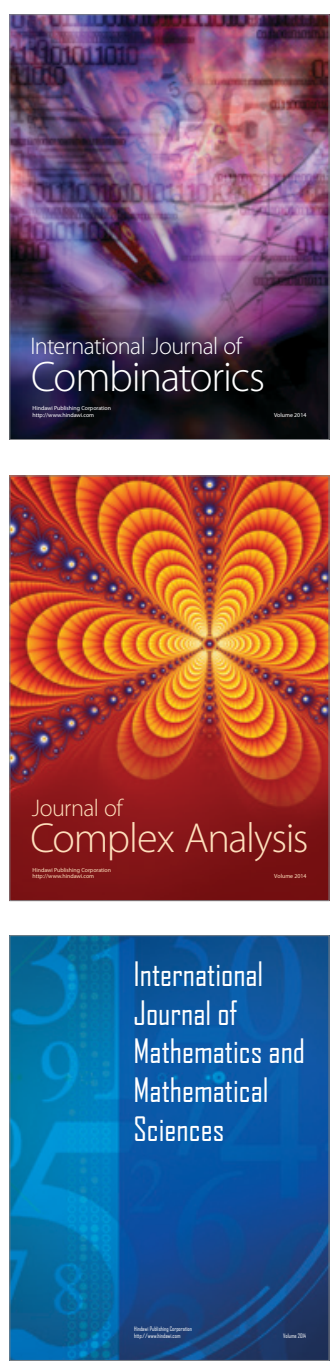
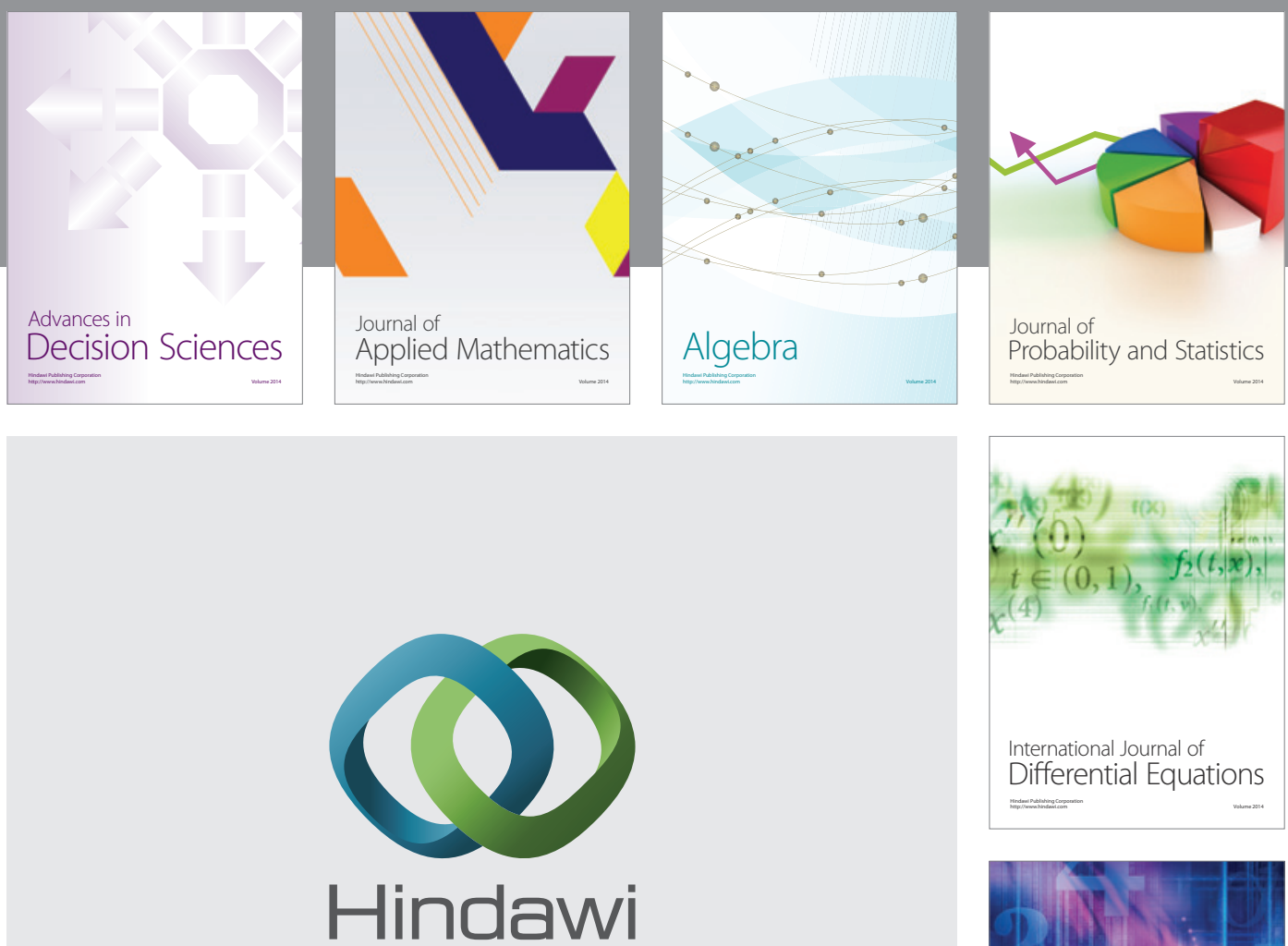

Submit your manuscripts at http://www.hindawi.com
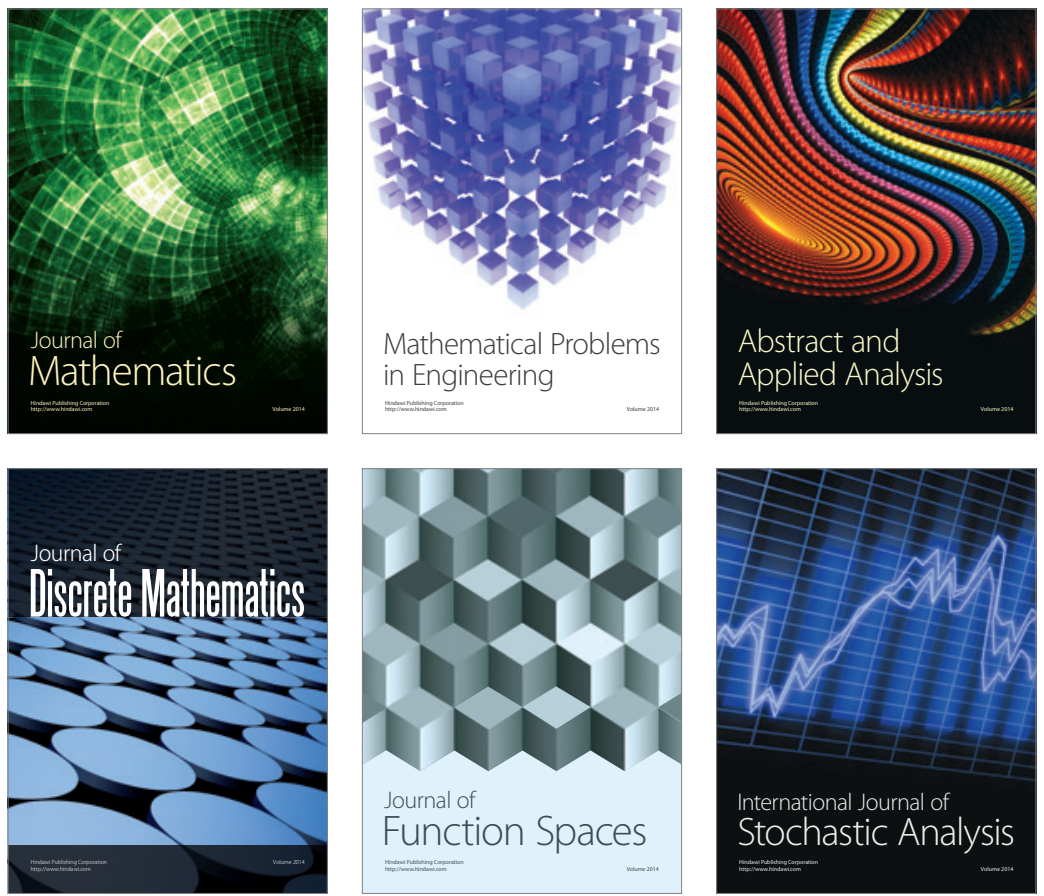

Journal of

Function Spaces

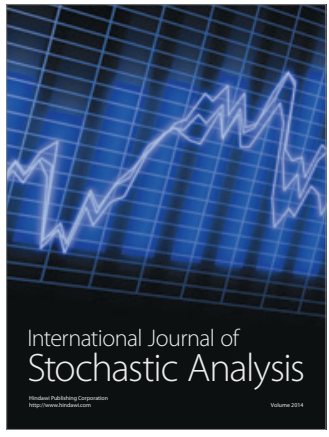

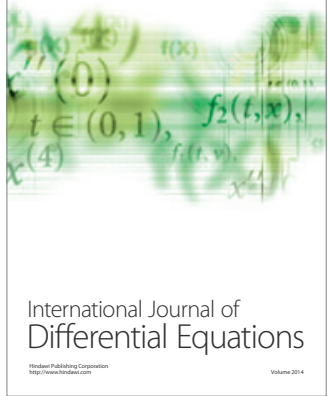
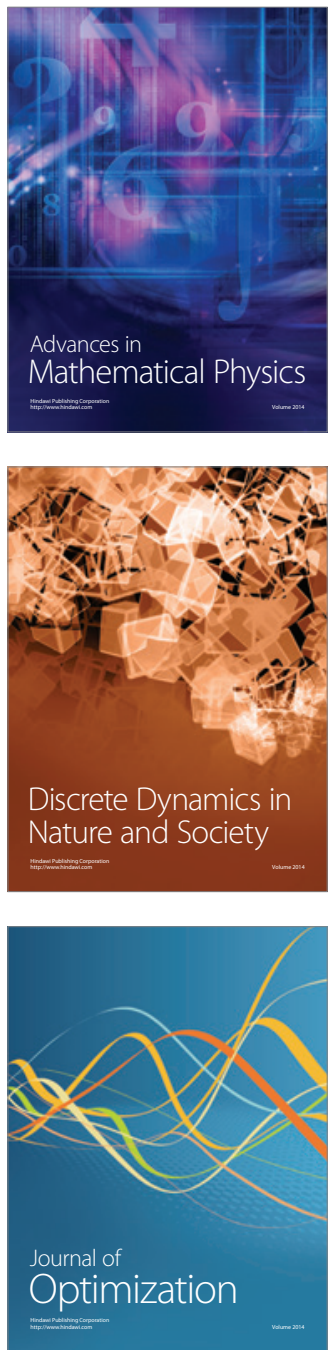